\title{
Low complexity predistortion and equalization in nonlinear multicarrier satellite communications
}

\author{
Efrain Zenteno ${ }^{1,2^{*}}$, Roberto Piazza ${ }^{3}$, Bhavani Shankar Mysore Rama Rao ${ }^{3}$, Daniel Rönnow ${ }^{1}$ \\ and Björn Ottersten ${ }^{3}$
}

\begin{abstract}
Aiming to reduce the power/mass requirements in satellite transponders and to reduce mission costs, joint amplification of multiple carriers using a single high-power amplifier (HPA) is being considered. In this scenario, a careful investigation of the resulting power efficiency is essential as amplification is nonlinear, and multicarrier signals exhibit enlarged peak-to-average power ratio. Thus, operating the amplifier close to saturation vastly increases signal distortion resulting in a severe degradation of performance, especially for higher order modulations. This paper proposes a reduced-complexity digital predistortion (DPD) scheme at the transmitter and a corresponding equalizer $(E Q)$ at the receiver to mitigate these nonlinear effects. Scenarios include both the forward as well as the return links. In particular, the paper exploits the MIMO Volterra representation and builds on a basis pursuit approach using a LASSO (least absolute shrinkage and selection operator) algorithm to achieve an efficient basis representation, avoiding large computational complexity, to describe the selection of predistorter/equalizer model. The work further compares and contrasts the two mitigation techniques taking various system aspects into consideration. The gains in performance and amplification efficiency demonstrated by the use of DPD/ EQ motivate their inclusion in next-generation satellite systems.
\end{abstract}

Keywords: Satellite communications; Multicarrier signal; MIMO systems; Joint amplification; Nonlinear distortions; Predistortion DPD; Equalization; LASSO

\section{Introduction}

Similar to its terrestrial counterpart, there is an increase in the demand for higher data rates and spectral efficiency in satellite communications. In fact, the rapid improvement in terrestrial data rate offerings has had a major role in this increased demand. Recent examples of this trend are the KA-Sat with a capacity of about 70 GBps and Viasat- 1 that reaches a total of $140 \mathrm{GBps}$ (the highest capacity broadcast satellite till date).

In a typical satellite broadcast system, the data stream is processed by three entities: the gateways, the satellite transponder, and the end-user terminals. The gateways

\footnotetext{
${ }^{*}$ Correspondence: Efrain.Zenteno@hig.se

1 Department of Electronics, Mathematics and Natural Sciences, University of

Gävle, Kungsbäcksvägen 47, 801-76 Gävle, Sweden

2 Department of Signal Processing, The Royal Institute of Technology KTH,

Osquldas väg. 10, 100-44 Stockholm, Sweden

Full list of author information is available at the end of the article
}

transmit the data stream in a form suitable for reception by a satellite. Transparent payloads, where the uplink data is mainly frequency shifted, amplified, and forwarded to users, are by far the most common telecom satellite architectures due to their competitive cost and technological flexibility. Clearly, in such payloads, the signal processing carried out on the ground can be updated based on technological advances in the course of the lifetime of the satellite. Each satellite transponder receives the data signal from one or more gateways and processes it before redirecting it to the ground receivers. In the widespread direct-to-home (DTH) services, the end receivers are fixed integrated receiver decoders [1] for TV applications.

To ensure that the amplification is power efficient, the high-power amplifiers (HPAs) are operated close to the saturation point. However, these HPAs suffer from nonlinear effects when driven close to saturation leading to signal distortion [2]. High order signaling/modulation

\section{是 Springer}

(c) 2015 Zenteno et al: licensee Springer. This is an Open Access article distributed under the terms of the Creative Commons Attribution License (http://creativecommons.org/licenses/by/4.0), which permits unrestricted use, distribution, and reproduction in any medium, provided the original work is properly credited. 
techniques, such as 16/32 amplitude and phase shift keying (APSK), are often used to increase spectral efficiency in the Digital Video Broadcasting - Satellite - Second Generation (DVB-S2) system [3]. However, these modulation schemes are sensitive to the nonlinear distortions introduced by the onboard HPA. This leads to a tradeoff between power and spectral efficiency. The nonlinear effects of the HPA become even more prominent when multiple carriers are amplified using a single HPA [4,5]. Such a situation arises when different carriers share the same onboard HPA due to power/mass and flexibility requirements. Joint amplification allows sharing of satellite resources among different links to reduce the mission cost. However, this operation leads to spurious signal components arising due to the inter-modulation products (IMD) and adjacent channel interference (ACI) caused by the HPA nonlinearity. Additionally, the use of multiple carriers leads to high peak-to-average power ratios, which requires an increase in the back-off used for power amplification, thereby leading to a loss in power efficiency. These effects are manifested as spectrum-inefficient frequency carrier segregation and power loss. Apart from amplification, the payload forwards or channelizes the data from the gateway to the respective users. This involves filtering which causes intersymbol interference (ISI) that further degrades the performance.

In order to improve both power and spectral efficiency, countermeasures have to be put in place. Transmitter techniques, known as digital predistortion (DPD), pre-process the signal with an equivalent inverse channel function to reduce the generated interference while receiver equalization (EQ) performs traditional interference cancellation [6]. Mitigation techniques usually have different goals. Compared to terrestrial telecommunications where DPD techniques mainly aim to suppress the out-of-band emissions [7], satellite communications aim to reduce in-band signal distortion $[8,9]$ since the out-ofband emissions are cut-out by the onboard multiplexing filter following the HPA in the satellite transponder.

Most mitigation techniques can be classified into three categories: model, neural network, and look-up table (LUT). In model-based mitigation techniques, the use of Volterra models [10] or pruned Volterra basis is extended $[7,11]$. Neural network mitigation approaches have been reported for SISO (single carrier) in satellite $[12,13]$ and terrestrial applications $[14,15]$. On the other hand, MIMO neural networks are usually sensitive to the training data and due to their complexity they are not extensively used in mitigation techniques that require the compensation of dynamic effects [16]. Look-up tables for mitigation of SISO (single carrier) systems appeared in satellite [2] and in its terrestrial counterpart [11]. However, MIMO (multicarrier) LUTs are still in early stages of research [17]. In general, MIMO LUTs have large amount of entries, which make their population cumbersome and increase their computational resources compared to SISO LUTs [18]. Due to the different nature of the mitigation techniques and for the sake of clarity, this work compares only model-based mitigation techniques.

The mitigation techniques can be applied at different stages; in terrestrial applications, they usually operate at the waveform domain and hence at a higher sampling rate, commonly called signal DPD [11,19]; while in satellite applications, the common practice is to apply them at symbol sampling rate and are thus denoted as data DPD/EQ $[8,20]$. Signal DPD can be applied to a single carrier (SISO) scenario or to single signal that is a superposition of multiple carriers. A number of papers have dealt with the former [11], while [19] has applied signal DPD to the latter scenario. The application of signal DPD on the superposition of multiple carriers can require very wide band processing, and hence DPD of the individual carriers in a multiple carrier scenario has been suggested to reduce the requirements on hardware and processing capabilities [21]. Multicarrier (MIMO) signal DPD has been studied in terrestrial applications for multiple channel transmitters $[22,23]$ and concurrent dual band amplifiers [24]. While terrestrial applications imply colocation of the predistorter and the HPA, the same is not the case in the envisaged multiple carrier scenario in satellite communications, and hence refer to as remote predistortion [25]. Particularly, the predistorter is located at the GW, the HPA is onboard the satellite and the uplink is regulated by spectral mask. Further, the tight constraints on the spectral mask of the uplinked satellite signal were naturally not considered in [22-24] where spectral regrowth was generated by the signal DPD. The uplink bandwidth restriction has motivated the multicarrier data DPD for satellite HPAs in [5] where a general multicarrier memory polynomial data predistorter algorithm was formulated. On the other hand, nonlinear iterative interference cancellation EQ techniques have been proposed in the area of satellite communications in $[6,26]$ for single carrier applications, while in [4] a turbo equalization method is provided for multicarrier satellite channels. In common broadcast applications involving multicarrier systems, the gateway $(\mathrm{GW})$ has access to all carriers while the user terminal can decode only one carrier. This scenario allows joint processing of all carriers only at the GW which enables multicarrier DPD [5] for interference mitigation. However, as anticipated in the next generations of satellite systems, joint processing of all carriers can still be envisaged enabling the use of multicarrier EQ techniques as described in $[4,8]$. Such scenarios include professional receivers on the forward link having enhanced capability or GW processing of multiple carriers on the reverse link with carriers originating from different users. The latter scenario arises since the return link comprises elements 
similar to forward link described earlier [8] and it excludes the possibility of a joint DPD.

In this work, we design low complexity mitigation techniques for multicarrier nonlinear satellite channels working at the symbol rate (data level). The reason for using data level instead of signal level in the mitigation techniques is twofold: first, data level operation does not introduce a spectral regrowth in the predistorted signal as required for the satellite regulatory authority [3]. Secondly, the inclusion of DPD in an early stage in the communication system (as data level) reduces the computational resources required for the mitigation technique while simultaneously enable the DPD deployment in scenarios where the signal level is not accessible or available, for instance, in carrier transmissions from different gateways.

We compare two distinct scenarios: the first one where only joint processing is applicable at the GW employing DPD [5]; secondly, we consider a scenario where joint processing of all carriers can be performed at the receiver employing equalization [8]. Building on the works of multicarrier data DPD [5] and equalization [4], we provide a solution that minimizes the complexity of the implementation with minor performance degradation. Complexity reduction is obtained by systematically reducing the MIMO Volterra basis function set using the LASSO (least absolute shrinkage and selection operator), algorithm [27] in a basis pursuit approach. The solution obtained by such an algorithm is sparse, producing an efficient basis representation of the system. While, a similar algorithm has been applied to reduce complexity in Volterra models [28], to the best of the authors' knowledge this is the first time a LASSO algorithm is used in a multicarrier framework. The multicarrier scenario deals with a much larger base set compared to the single carrier system, thereby strongly motivating the application of our approach. In this paper, we also trade-off the predistorter and equalizer for performance, complexity, and ease of implementation, with emphasis on parameter identification in time-varying channels.

\section{Multiple carrier transmission: system overview Scenario}

The system considered involves broadcast transmission from a single gateway to many receivers through a transparent satellite transponder wherein only filtering to remove out-of-band noise, amplification, and channelization of the streams are assumed to occur. The transmission is in the $\mathrm{Ku}$ band (in Europe, $\mathrm{Ku}$ band is used from 10.7 to $12.75 \mathrm{GHz}$ ) reflecting the state-of-the-art in satellite operations [3]. However, the proposed methodology is general to multicarrier links and the mentioned frequency band has no impact on it.

Figure 1 illustrates the system under consideration which will be further detailed in the sequel. The GW transmits $K$ carriers to the satellite; where the $i$ th carrier is upconverted to a center frequency $f_{i}$ after pulse shaping with $p_{i}(\cdot)$. The bandwidth of each carrier is set depending on the total number of carriers and the transponder bandwidth (typically $36 \mathrm{MHz}$, but it could be as large as $500 \mathrm{MHz}$ ). In this study, all the carriers have the same bandwidth and are assumed to be compliant with DVB-S2 specifications.

The signals from the GW are channelized to the satellite HPA through a wideband input multiplexing (IMUX) filter. Typical amplitude and group delay response of such filters can be obtained from [3]. Traveling wave tube amplifiers (TWTAs) are the HPAs predominantly used onboard and are intrinsically nonlinear. Further, the TWTAs used in Ku band can be assumed to have a transfer characteristic largely independent of the frequency [20]. Subsequently, the output multiplexing (OMUX) filter is used to reduce the out-of-band emission before relaying the HPA output to the antenna sub-system.

From a system perspective, the DPD needs knowledge of the channel characteristics in terms of filters, amplifiers, etc. While this can be obtained prior to launch, the quasi-static nature of the channel, which is due to diurnal changes and ageing, requires information about the channel at regular intervals during operation. Towards this, we assume the existence of dedicated receivers, called

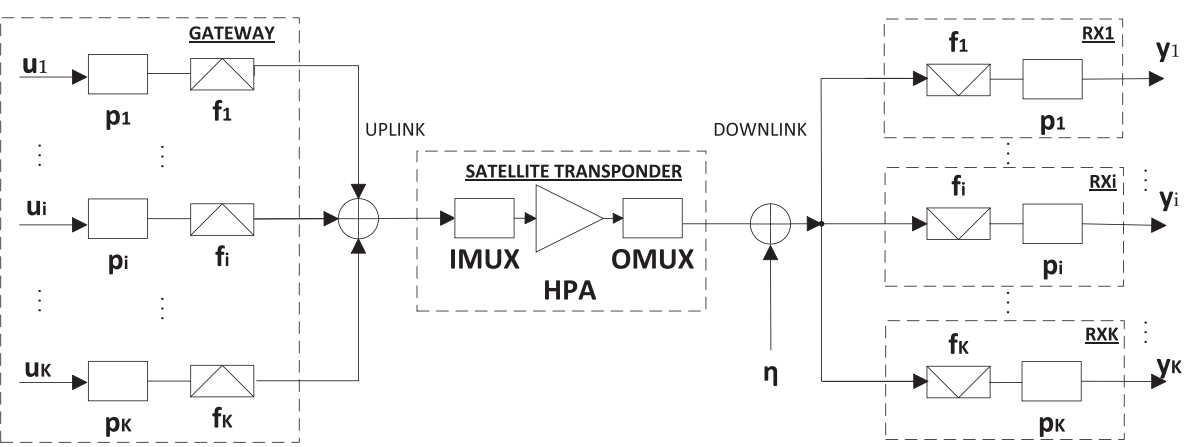

Figure 1 Satellite system architecture supporting $K$ carriers amplified simultaneously by a single onboard HPA. 
reference terminals, which are capable of multicarrier processing, whose function is to provide channel estimates for calibration. Such receivers are operator managed and can have much better noise figures compared to commercial user terminals.

\section{Signal and channel model}

Let $u_{i}(n)$ be the $n$th transmitted symbol in the $i$ th carrier. These symbols are pulse shaped with $p_{i}(t)$ yielding the complex-valued base-band signal,

$$
s_{i}(t)=\sum_{n} u_{i}(n) p_{i}\left(t-n T_{r}\right) .
$$

$T_{r}$ is the symbol duration, commonly referred to as the symbol rate. In this work, we consider $p_{i}(t)$ a squareroot raised cosine filter with roll-off factor $\rho$ in accordance with the DVB-S2 standard [3]. Each one of the $K$ base-band signals is upconverted to a carrier frequency $f_{i}$ and added together. Thus, rendering the pass-band representation,

$$
s(t)=\sum_{i=1}^{K} \operatorname{Re}\left\{s_{i}(t) \mathrm{e}^{j\left(2 \pi f_{i} t+\varphi_{i}\right)}\right\},
$$

where $\varphi_{i}$ is the phase of the $i$ th carrier used for upconversion, $j=\sqrt{-1}$, and $\operatorname{Re}\{\cdot\}$ denotes the real part of the complex argument.

The satellite transponder is formed by a cascade of IMUX, HPA, and the OMUX. The bandpass filters (IMUX/OMUX) are modeled as finite impulse response (FIR) digital filters. The HPA is characterized by the amplitude and phase distortion curves, known as $\mathrm{AM} / \mathrm{AM}$ and $\mathrm{AM} / \mathrm{PM}$ curves, and are modeled by the well-known Saleh model [29]. The model is described in Equation 3, and the parameters of the model for a typical DVB-S2 application can be obtained by curve fitting to the experimental data in [3],

$$
\begin{aligned}
& A(z)=\frac{\alpha_{0} z}{1+\alpha_{1} z^{2}}, \\
& \Gamma(z)=\frac{\beta_{0} z^{2}}{1+\beta_{1} z^{2}}
\end{aligned}
$$

with $z$ denoting the magnitude of the complex-valued base-band signal at the input of the HPA, and $A, \Gamma$ representing its $\mathrm{AM} / \mathrm{AM}$ and $\mathrm{AM} / \mathrm{PM}$ conversion, respectively.

Based on the HPA and IMUX/OMUX described above, the satellite transponder can be modeled as a nonlinear system with memory. The memoryless nonlinearity causes constellation warping and ACI while the ISI is caused by the filters per se and their coupling with the nonlinearity. While ISI and constellation warping are present in a single carrier system [2], multicarrier systems are further affected by ACI and IMD that severely distort the received symbols [4].
The output of the transponder is received at the terminals perturbed by additive white Gaussian noise (AWGN). The receiver employs a filter matched to the pulse shaper $p_{i}(t)$. After matched filtering and sampling, the received symbols on the $i$ th carrier take the form,

$$
y_{i}(n)=g_{i}\left(\left\{\left\{u_{i}(n-l)\right\}_{l=0}\right\}_{i=1}^{K}\right)+\eta_{i}(n)
$$

where $g_{i}(\cdot)$ models the transponder effect on the $i$ th carrier and $\eta_{i}(n)$ is the AWGN. Note that the received symbols corresponding to the $i$ th carrier are a function of all carrier sequences through $g_{i}(\cdot)$. In general, the model $g_{i}(\cdot)$ includes the time and phase delay of the channel. The phase delay contribution appears naturally in a multicarrier scenario where the central frequency of a carrier does not coincide with the central frequency of the pass band of the filters involved. To keep the study focused on nonlinear effects, both time and phase delay are ideally compensated in the receiver, thus not influencing the performance. In the following, we develop a model of $g_{i}(\cdot)$ using the MIMO Volterra series.

\section{Volterra analysis}

A time invariant nonlinear dynamic system with fading memory can be described by Volterra theory [30]. This theory has been extended to multiple input multiple output systems (MIMO) in [31-33]. However, it is convenient to describe the Volterra series in discrete complex baseband representation as in [6,34] since communication signals are denoted and manipulated in this domain. The continuous time MIMO Volterra [31-33] and the complex base-band formalism [34] were combined in [23], in which a complex $2 \times 2$ Volterra system was formulated. Extending that results to an arbitrary number of carriers, we get:

$$
\begin{aligned}
& y_{i}(n)=y_{i}^{(1)}(n)+y_{i}^{(3)}(n)+y_{i}^{(5)}(n)+\ldots \\
& y_{i}^{(1)}(n)=\sum_{k_{1}=1}^{K} \sum_{m_{1}=0}^{\infty} h_{k_{1}}\left(m_{1}\right) u_{k_{1}}\left(n-m_{1}\right), \\
& y_{i}^{(3)}(n)=\sum_{k_{1}, k_{2}, k_{3}=1}^{K} \sum_{m_{1}, m_{2}, m_{3}=0}^{\infty} h_{k_{1}, k_{2}, k_{3}}\left(m_{1}, m_{2}, m_{3}\right) \ldots \\
& u_{k_{1}}\left(n-m_{1}\right) u_{k_{2}}\left(n-m_{2}\right) u_{k_{3}}^{*}\left(n-m_{3}\right), \\
& y_{i}^{(5)}(n)=\sum_{k_{1}, \ldots, k_{5}=1}^{K} \sum_{m_{1}, \ldots, m_{5}=0}^{\infty} h_{k_{1}, \ldots, k_{5}}\left(m_{1}, \ldots, m_{5}\right) \ldots \\
& u_{k_{1}}\left(n-m_{1}\right) u_{k_{2}}\left(n-m_{2}\right) u_{k_{3}}\left(n-m_{3}\right) \ldots \\
& u_{k_{4}}^{*}\left(n-m_{4}\right) u_{k_{5}}^{*}\left(n-m_{5}\right),
\end{aligned}
$$


where $i=1, \ldots, K \cdot u_{i}(n)$ and $y_{i}(n)$, respectively, denote the transmitted and received symbols on the $i$ th carrier at $n$th instance. Further, the signal $y_{i}^{(p)}(n)$ represents the contribution of the $p$ th nonlinear order at the $i$ th received carrier, and $\left\{h_{k_{1}}, \ldots\left(m_{1}, \ldots\right)\right\}$ are the Volterra kernels [30]. Note that Equation 5 only includes odd nonlinear orders since those are responsible for the in-band distortion encountered at the output of the nonlinear channel [34]. For complexity reasons to be discussed below and for fostering a hardware implementation, the series in Equation 5 is truncated to a certain memory depth and nonlinear order.

Assuming that the series (Equation 5) is truncated to $P$ th nonlinear order with $M_{p}$ being the memory depth for the $(2 p-1)$ th nonlinear order, the number of parameters in Equation 5 corresponds to $\sum_{p=1}^{\frac{p+1}{2}} K^{2 p-1}\left(M_{p}+1\right)^{2 p-1}$. This number exponentially increases with nonlinear order and memory depth and further motivates the truncation. Figure 2 illustrates this relationship using $M_{p}=2$ for all nonlinear orders. The number of terms grows with the nonlinear order $(P)$, memory depth $\left(M_{p}\right)$, and the number of carriers $(K)$. Notice that the number of parameters for the MIMO system are orders of magnitude larger than for the SISO system. Even with a modest nonlinear order of 3 , a $5 \times 5$ MIMO system has 100 times more parameters than the corresponding SISO system. The large number of parameters in the latter is a well-known problem and has given rise to large research efforts in finding more parameter-efficient models [11]. There is a certain level of redundancy in the formulation due to the permutations of the terms that can lead to identical contribution, such as, $x_{i} x_{j} x_{z}^{*}=x_{j} x_{i} x_{z}^{*}$, referred as symmetry [33]. The general trends are, however, the same as presented in Figure 2 even if redundancy is considered.

The Volterra model in Equation 5 describes the impairments in the received carrier symbols; for every nonlinear order $p$. These terms can be further identified as IMD, ISI, and $\mathrm{ACI}$ that limit the achievable throughput. Enhancing the throughput in such situations is taken up next.

\section{Nonlinear mitigation techniques}

In this section, we describe the two designed countermeasures for multicarrier nonlinear channels: DPD and EQ. The parameter identification method applied to both techniques is presented, and, subsequently, we provide the method for complexity reduction based on a basis pursuit approach.

\section{Multicarrier data predistortion}

Digital predistortion (DPD), introduced at the GW, aims to mitigate the channel interference and to increase power efficiency. Joint processing of carriers allows precancellation of the relevant interference generated by the IMD products. Further, processing is performed at data level (at symbol rate), prior to pulse shaping, in order to avoid signal spectral regrowth on the uplink channel

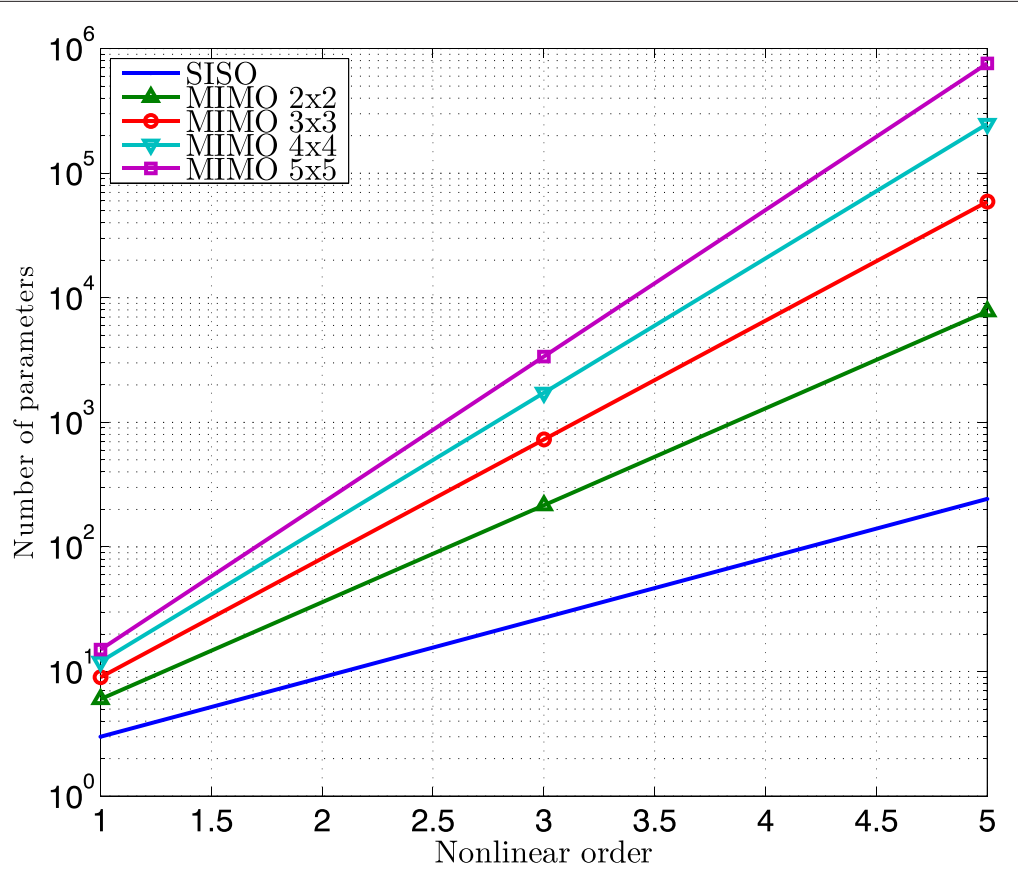

Figure 2 Number of parameters versus the nonlinear order in the Volterra series using $\mathbf{2}$ memory depth for all nonlinear orders. Single (SISO) and multiple input/output (MIMO) channels are reported. Redundancy due to the symmetry was not considered. 
and to reduce the GW hardware requirements [21] (cf. Figure 3).

The predistorter function is designed to approximate the inverse channel, which is also nonlinear and dynamic, and hence can be described by Volterra series. As a consequence, the predistorter output for the $i$ th carrier, $q_{i}(n), 1 \leq i \leq K$ can be described as,

$$
q_{i}(n)=\left[\boldsymbol{\phi}_{i}\left(\boldsymbol{u}_{M}(n)\right)\right]^{T} \boldsymbol{w}_{i},
$$

where $q_{i}(n)$ denotes the predistorted symbols and $\boldsymbol{w}_{i}$ is the $\sum_{p=1}^{\frac{P+1}{2}} K^{2 p-1}\left(M_{p}+1\right)^{2 p-1} \times 1$ vector of the predistorter parameters which are the coefficients of the series (Equation 5). Thus, the number of parameters in $\boldsymbol{w}_{i}$ corresponds to the number of basis functions of the MIMO Volterra series. $\phi_{i}()$ is the vector comprising the nonlinear input combinations. In particular, we define,

$$
\begin{aligned}
\boldsymbol{u}(n) & =\left[u_{1}(n), \ldots, u_{K}(n)\right]^{T}, \\
\boldsymbol{u}_{M}(n) & =\left[\boldsymbol{u}^{T}(n-M), \ldots \boldsymbol{u}^{T}(n+M)\right]^{T} .
\end{aligned}
$$

Further, the entries of the $\sum_{p=1}^{\frac{P+1}{2}} K^{2 p-1}\left(M_{p}+1\right)^{2 p-1} \times 1$ vector $\phi_{i}()$ are the Volterra basis $\phi_{k_{1}, \ldots, k_{d} / m_{1}, \ldots, m_{d}}^{\{d\}}$ defined as [34],

$$
\begin{aligned}
\phi_{k_{1}, \ldots, k_{d} / m_{1}, \ldots, m_{d}}^{\{d\}}(\boldsymbol{u}(n))= & \prod_{j=1}^{(d+1) / 2} u_{k_{j}}\left(n-m_{j}\right) \ldots . \\
& \prod_{j=(d+1) / 2+1}^{d} u_{k_{j}}^{*}\left(n-m_{j}\right) .
\end{aligned}
$$

For each nonlinear order $d$, we stack terms relative to all carrier combinations together with memory combinations in the $K^{2 d-1}\left(M_{d}+1\right)^{2 d-1} \times 1$ vector

$$
\boldsymbol{\phi}_{i}^{\{d\}}\left(\mathbf{u}_{M_{d}}(n)\right)=\left[\left\{\phi_{k_{1}, \ldots, k_{d} / m_{1}, \ldots, m_{d}}^{\{d\}}(\boldsymbol{u}(n))\right\}\right],
$$

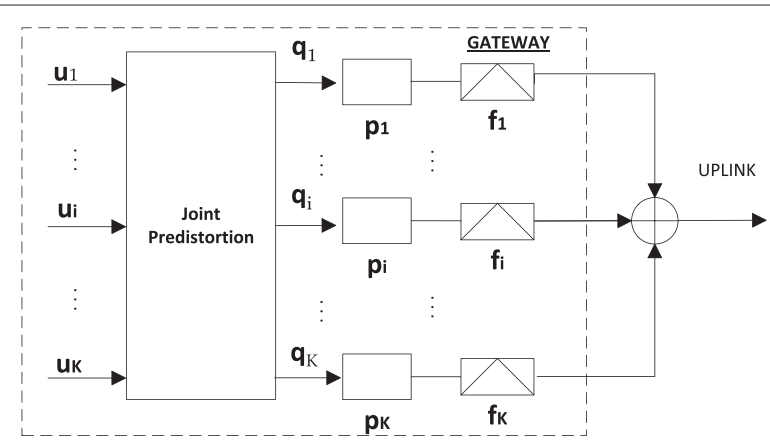

Figure 3 Multicarrier predistortion (DPD) architecture: $K$ carriers are simultaneously processed at the GW. with $k_{j} \in(1, K), m_{j} \in\left(-M_{d}, M_{d}\right)$ and $d$ denoting the nonlinear order considered. Finally, we augment vectors of different nonlinear orders obtaining,

$$
\boldsymbol{\phi}_{i}\left(\mathbf{u}_{M}(n)\right)=\left[\left\{\boldsymbol{\phi}_{i}^{\{d\}}\left(\mathbf{u}_{M_{d}}(n)\right)\right\}\right],
$$

with $d \in(1, P), \boldsymbol{\phi}_{i}\left(\mathbf{u}_{M}(n)\right)$ being a $\sum_{p=1}^{\frac{P+1}{2}} K^{2 p-1}\left(2 M_{p}+\right.$ $1)^{2 p-1} \times 1$ vector. Identification of the parameters $\boldsymbol{w}_{i}$ is performed such that the predistorter function approximates the channel post-inverse. This can be achieved using the indirect learning architecture [10] where the inverse is estimated from the input and output signals. A detailed description of the parameter identification method is provided in the sequel. The number of basis functions becomes very high, as described previously; we therefore reduce their number while retaining the model performance as described below.

\section{Multicarrier data equalization}

From a system point of view, a completely different approach would be to compensate for the nonlinear interference at the receiver side. Joint processing of multicarrier signals at the user terminals can be envisaged in broadcast applications where the receiver can decode more than one carrier (professional application, for example). Such receivers are expensive with the complexity and cost compared to user receivers being dictated by the market. Another application of multicarrier equalization is on the return link. Albeit, the return link in consumer applications is usually large making equalization complex. The setup is dual of the considered scenario, where different users uplink carriers that are simultaneously amplified by the onboard HPA and processed jointly at the GW. Complexity and cost of the user receivers are also tightly limited by the market.

Multicarrier equalization for nonlinear satellite channels was first introduced in [8] for a dual carrier channel. In [8], the authors designed and compared two different kinds of equalization techniques: a Volterra equalizer implementing the channel inverse function and an interference canceler based on the channel function identification. Results show that the interference canceler slightly outperforms the channel inversion approach. In [4], the interference cancellation method is further extended to a turbo Volterra architecture designed for an arbitrary number of carriers. In this work, we extend the dual carrier Volterra equalizer implementing the channel inverse [8] to an arbitrary number of carriers. The goal of our exercise is to reduce the receiver architecture complexity compared to [4] with minor performance degradation.

The considered equalization architecture is illustrated in Figure 4 and is described by,

$$
r_{i}(n)=\left[\boldsymbol{\phi}_{i}\left(\boldsymbol{y}_{M}(n)\right)\right]^{T} \boldsymbol{w}_{i},
$$




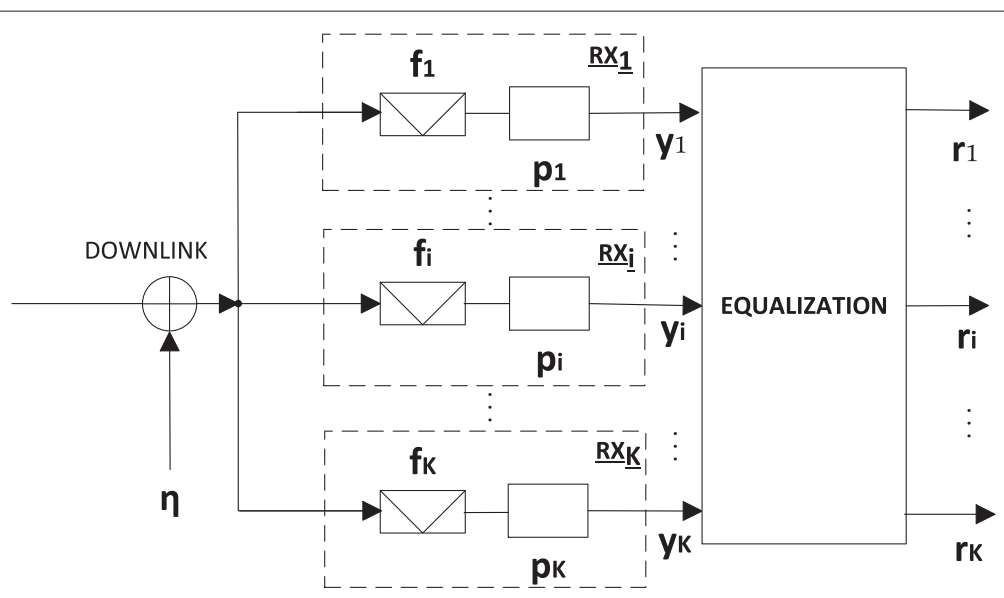

Figure 4 Multicarrier equalization (EQ) architecture: $K$ carriers are simultaneously processed at the receiver.

where $r_{i}(n)$ denotes the equalized symbols and $\boldsymbol{\phi}_{i}()$ is defined similarly to Equation 6 taking $\left\{y_{k}(n)\right\}_{k=1}^{K}$ as inputs. Following [8], we estimate the equalizer parameters $\boldsymbol{w}_{i}$ to implement the channel post-inverse function. In the following section, we provide the general formulation for channel post-inverse parameter identification that is valid for both DPD and EQ design.

\section{Identification of channel inverse}

From Equations 6 and 12, both the predistorter and the equalizer are described through linear model structures. Hence, the coefficients $\boldsymbol{w}_{i}$, denoting both the equalizer and the predistorter weights, can be estimated using linear techniques. While the aforementioned problem is formulated in a straightforward manner for equalizer based on Equation 12, it relates to the indirect learning architecture [10] for predistorter design. In the indirect learning architecture, the predistorter weights are obtained using the post-inverse (equalization) solution. In particular, we consider,

$$
u_{i}(n)=\left[\boldsymbol{\phi}_{i}(\boldsymbol{y}(n))\right]^{T} \boldsymbol{w}_{i}
$$

Given a dedicated training sequence providing $N$ samples of transmitter and received symbols, $\left\{u_{i}(n)\right\}_{i=1}^{K}$ and $\boldsymbol{y}(n)=\left[y_{1}(n), \ldots, y_{K}(n)\right]^{T}$, respectively, we can stack the quantities to obtain,

$$
\begin{gathered}
\boldsymbol{s}_{i}=\left[u_{i}(0) \ldots u_{i}(N-1)\right]^{T}, \\
\boldsymbol{\Phi}_{i}=\left[\begin{array}{c}
\boldsymbol{\phi}_{i}^{T}(\boldsymbol{y}(0)) \\
\vdots \\
\boldsymbol{\phi}_{i}^{T}(\boldsymbol{y}(N-1))
\end{array}\right] .
\end{gathered}
$$

From Equation 13, using Equations 14 and 15, it follows that $\boldsymbol{s}_{i}=\boldsymbol{\Phi}_{i} \boldsymbol{w}_{i}$. The least squares solution for $\boldsymbol{w}_{i}$ can then be readily obtained from [35] as,

$$
\boldsymbol{w}_{i}=\left(\boldsymbol{\Phi}_{i}^{H} \boldsymbol{\Phi}_{i}\right)^{-1} \boldsymbol{\Phi}_{i}^{H} \boldsymbol{s}_{i},
$$

where $H$ denotes the Hermitian transpose operator. The identified parameters can be equivalently used for DPD and EQ architectures. The large number of parameters in $\boldsymbol{w}_{i}$ makes the implementation of these techniques difficult and computationally expensive in practical applications. In the following section, we describe a method for substantially reducing the complexity of the model.

\section{Complexity reduction}

The parameters $\boldsymbol{w}_{i}$, representing the predistorter or equalizer coefficients (cf. (16)), are basis functions of a Volterra series (of the form of Equation 5) in which the input and output have been interchanged to describe the postdistorter function. The parameters $\boldsymbol{w}_{i}$ are commonly estimated using least squares identification methods. A system modeled by the Volterra series can be represented in multiple forms. Thus, different identification methods provide different system representations. Least square methods yield dense system representations, that is, most of the coefficients have significant weight in the model output. Hence, an implementation of the Volterra series becomes complex and its analysis cumbersome. A different approach followed by compressed sensing methods allows to reduce the number of coefficients while retaining the modeling properties. Such an approach is known as LASSO [27]; in the view of the principle of parsimony [36], such sparse model representations must be preferred. These techniques have been used successfully in SISO Volterra basis selection and polynomial models $[28,37,38]$.

The large number of coefficients of the predistorter or equalizer limits its applicability to only low nonlinear orders, short memory depth, and a few carriers. It is therefore, in practice, necessary to reduce the complexity by selecting the basis functions (coefficients) that are the most significant when reducing the model error. We use a form of the LASSO technique [27] similar to [39] 
to select the significant coefficients of the system. The LASSO solves a least squares problem constraining the model to be sparse; such a constraint is usually represented as a sum of the magnitude of the model parameters,

$$
\begin{array}{ll}
\underset{\left\{\boldsymbol{w}_{i}\right\}_{i}}{\operatorname{minimize}} & \frac{1}{N} \sum_{i=1}^{K}\left\|\boldsymbol{s}_{i}-\boldsymbol{\Phi}_{i} \boldsymbol{w}_{i}\right\|_{2}, \\
\text { subject to } & \sum_{n=1} R_{i, n}\left|w_{i}(n)\right| \leq \gamma_{i}, \quad i=1, \ldots, K .
\end{array}
$$

Here, $N$ is the total number of symbols used, the operator $\|\cdot\|_{2}$ denotes the $\ell_{2}$ norm, and $w_{i}(n)$ is the $n$th coefficient of the $\boldsymbol{w}_{i}$ vector

$$
\boldsymbol{w}_{i}=\left[w_{i}(1), w_{i}(2), \ldots, w_{i}\left(\sum_{p=1}^{\frac{p+1}{2}} K^{2 p-1}\left(M_{p}+1\right)^{2 p-1}\right)\right]^{T}
$$

$R_{i, n}$ is a scalar normalizing factor required since the coefficients in the predistorter and equalizer have different scales of magnitude provided by distinct nonlinear orders. $R_{i, n}$ is set as the sample variance of the $n$th column of the regression matrix $\boldsymbol{\Phi}_{i}$ or equivalently as the energy of the $n$th base function,

$$
R_{i, n}=\frac{1}{N} \varphi_{n}{ }^{H} \varphi_{n},
$$

where $\varphi_{n}$ denotes the $n$th column of the matrix $\boldsymbol{\Phi}_{i}$. Such a normalization is recommended for basis selection [40]. Despite Equation 17 is formulated similarly to the weighted LASSO [39], it does not compute the Ridge vector required for the constraint in the weighted LASSO.

In contrast to identification techniques using only the error square as a loss function, the solution of Equation 17 produces sparse solutions forcing some of the coefficients in $\boldsymbol{w}_{i}$ to reduce to zero and hence providing an efficient basis representation for the channel inverse. Note that the weight of each basis function is given by the corresponding coefficient in $\boldsymbol{w}_{i}$, and the estimated $\boldsymbol{w}_{i}$ is sparse. Hence, basis functions which corresponding coefficient is zero can be eliminated without sacrificing performance. The reduction in complexity from the use of this technique is shown in the next section.

\section{Simulations}

\section{Figures of merit}

The total degradation (TD) is used to evaluate the performance in coded satellite links. TD accounts for the nonlinear distortion while penalizing the loss in HPA power efficiency (measured as output power back-off $(\mathrm{OBO})$ ) [41],

$$
\mathrm{TD}_{i}={\frac{E_{s}}{N_{0} i}}^{N L}-{\frac{E_{s}}{N_{0} i}}^{L}+\mathrm{OBO}
$$

where $\frac{E_{s}}{N_{0}}{ }_{i} L$ and $\frac{E_{s} L}{N_{0}} i$ are the average symbol energy to noise ratios required to achieve a target bit error rate (BER) in the nonlinear channel and the ideal linear channel (AWGN), respectively, for the $i$ th carrier. While $\frac{E_{s}}{N_{o}}$ is a single carrier metric, the $\mathrm{OBO}$ depends on the combined signal and not on individual carriers (aggregate OBO). In all our simulations, we consider a target BER of $10^{-5}$. In contrast to equalization, the DPD will cause a change in the operating $\mathrm{OBO}$ of the satellite transponder due to the change in the signal statistics exciting the HPA. The OBO change, due to the use of DPD, is then included in the TD.

\section{Simulation settings}

The satellite transponder was simulated as the cascade of three systems: an IMUX filter, a TWTA, and an OMUX filter. The IMUX and OMUX filters were modeled by FIR structures using 51 and 41 taps, respectively, that operate at the simulation rate. The TWTA was modeled by the static nonlinear Saleh model in Equation 3 with $\alpha_{0}=2$, $\alpha_{1}=1, \beta_{0}=\frac{\pi}{6}$, and $\beta_{1}=1$. Table 1 summarizes the settings used in the simulations.

The simulation rate referred in Table 1 indicates the amount of upsampling used in the carrier signals. The upsampling is required to accurately represent the nonlinear effects. These effects cause the signal bandwidth to expand and is commonly referred as spectral regrowth. The LDPC coding scheme [42] in Table 1 is representative of the state of art in satellite communications [3].

\section{Basis selection results}

The system depicted in Figure 1 was simulated using Matlab, with the settings in Table 1 exploiting the builtin libraries for modulation and coding that are compliant

Table 1 Simulation settings

\begin{tabular}{llc}
\hline & & Three-carrier \\
\hline \multirow{3}{*}{ Signal } & Modulation format & 8 PSK, 16/32 APSK \\
& Symbol rate $\left(T_{r}\right)$ & 7 M Baud \\
& Carrier Spacing & $1.25 T_{r}$ \\
& Coding scheme & LDPC $\frac{3}{4}, \frac{4}{5}, \frac{5}{6}, \frac{8}{9}, \frac{9}{10}$ \\
\hline \multirow{3}{*}{ Channel } & Pulse-shaping filter & SRRC $\rho=0.25$ \\
& IMUX bandwidth & $26 \mathrm{MHz}$ \\
& OMUX bandwidth & $32 \mathrm{MHz}$ \\
& Simulation rate & 20 \\
& HPA & Saleh's model
\end{tabular}


with DVB-S2 [3]. The problem in Equation 17 was solved using convex solvers $[43,44]$.

The constraints $\gamma_{i}$ in Equation 17 must be adjusted properly to produce sparse/relevant basis functions. This can be addressed by plotting the Pareto optimal boundary for the problem, as depicted in Figure 5 ([45], Chapter 6). It is suggested to choose $\gamma_{i}$ at the knee point of the curve; this point provides a trade-off between number of basis functions and the goodness of fit of the model.

Note that in Figure 5, there is a region to the left in the figure where the model error (on the horizontal axis) is insensitive to variations of $\sum_{n} R_{i, n}\left|w_{i}(n)\right|$ (on the vertical axis). Since $\sum_{n} R_{i, n}\left|w_{i}(n)\right|$ is a metric for the model sparsity, by selecting $\gamma_{i}$ as suggested, it is possible to promote the model sparsity without compromising for the model error.

We test the Volterra model of Equation 5 for a $K=3$ carrier case with the LASSO algorithm to distinguish the relevant bases for the system inverse. The simulated system is described in Figure 1 with the parameters indicated in Table 1, using 16 APSK symbols in every carrier, the transponder aggregate $\mathrm{OBO}$ was set to $2 \mathrm{~dB}$, and the number of symbols used in the basis selection corresponds to a frame in DVB-S2 [3], that is, 64,800 symbols. Figure 6 shows the magnitude of the MIMO Volterra parameters rendered by the LASSO solver. The predistorters are shown in colors for each carrier. Figure 6 indicates some basis functions corresponding to the predistorter of carrier 3. The LASSO technique in Equation 17 does not produce coefficients with values equal to zero; instead very small values are rendered [46], as noted in Figure 6. Hence, we retained the basis functions with coefficient $\boldsymbol{w}_{i}$ larger than $10^{-4}$ in the model, and the rest were eliminated. Noted that from Figure 6 , the amplitude variations of the vector $\boldsymbol{w}_{i}$ is of several orders of magnitude. Thus, altering the threshold level of $10^{-4}$, used for discriminating the basis functions, did not change the basis selection results.

The Volterra basis model for the channel inverse was truncated to nonlinear order 5 and memory depths $M_{1}=$ 5, $M_{2}=3$, and $M_{3}=1$ ( 5 for the linear, 3 and 1 for the third and fifth nonlinear orders, respectively). This original set of bases is redundant when considering the compensation of distortion channel effects, as can be seen in the left part of Figure 5. The 'knee' shape in Figure 5 indicates possible parsimonious system representation for which no degradation of the model error is observed. The total number of tested basis functions is 1,458 when considering symmetric representation of Equation 5, from which only 17 basis functions are finally selected and reported in Table 2. Thus, 17 basis functions are used in the mitigation technique, which is formed as a linear combination of the basis indicated in Table 2. It is worthwhile noticing in the equally spaced carrier scenario, that the third-order nonlinear terms $x_{i} x_{j} x_{k}^{*}$ interfere to the $(i+j-k)$ th carrier. This is found out during the basis selection process as noted by the columns of Table 2. This 17 basis functions could be compared to the result for a multicarrier equalizer in [4], where at least 30 basis functions were selected by using a different approach that resorts to enumerating the contribution at certain frequencies.

The basis selection test was repeated for different modulation formats, code rates, and operating aggregate OBOs of the HPA; despite the varied settings, the basis

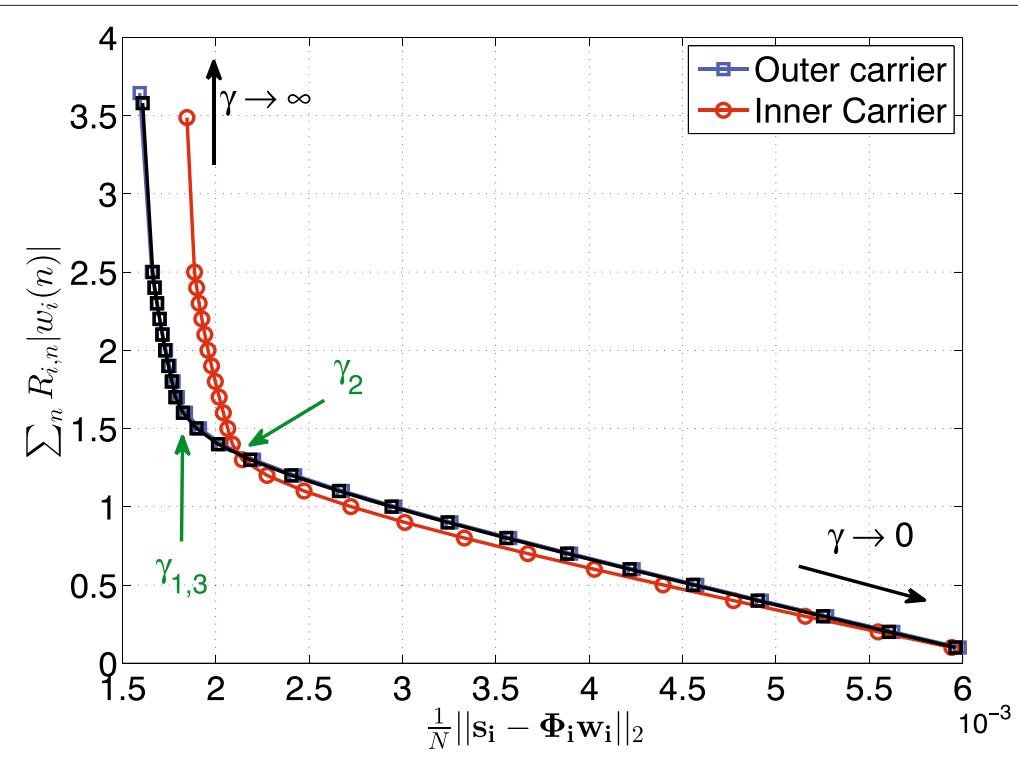

Figure 5 Weighted sum of the magnitude of the parameters versus the model error in a three-carrier simulation. The curves are obtained by sweeping $\gamma_{i}$. 


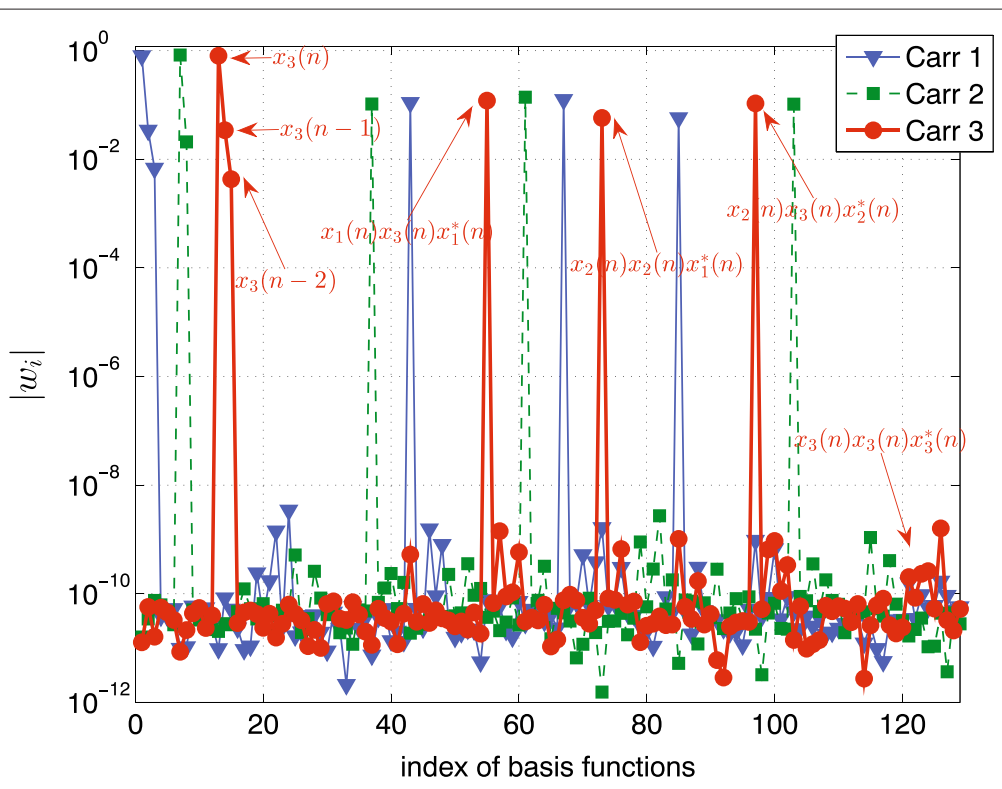

Figure 6 Magnitude of the parameters in the MIMO Volterra series when using the proposed LASSO solver. Reported 125 of the 1458 total parameters.

selection gives similar results to those reported in Table 2. These tests were however repeated under the following conditions:

- The carriers have the same frequency locations.

- The relative power between carrier signals is kept constant.

- The carriers have the same bandwidth (symbol rate $T_{r}$ is fixed).

- The IBO is varied only in the region where significant amount of nonlinear distortion is produced.

Obtaining the same basis representation, despite the varied settings, suggests that a parsimonious system representation can be found at data domain (symbol level) which is the cause of the observed behavior. From Table 2, larger memory is required for the outer carriers than the inner (central) carrier, as they are affected more by

Table 2 Selected basis functions for the system inverse in a three-carrier satellite link

\begin{tabular}{ccc}
\hline Carrier $\mathbf{1}$ & Carrier 2 & Carrier 3 \\
\hline$x_{1}(n)$ & $x_{2}(n)$ & $x_{3}(n)$ \\
$x_{1}(n-1)$ & $x_{2}(n-1)$ & $x_{3}(n-1)$ \\
$x_{1}(n-2)$ & - & $x_{3}(n-2)$ \\
\hline$x_{2}(n) x_{2}(n) x_{3}^{*}(n)$ & $x_{1}(n) x_{2}(n) x_{1}^{*}(n)$ & $x_{1}(n) x_{3}(n) x_{1}^{*}(n)$ \\
$x_{1}(n) x_{3}(n) x_{3}^{*}(n)$ & $x_{1}(n) x_{3}(n) x_{2}^{*}(n)$ & $x_{2}(n) x_{2}(n) x_{1}^{*}(n)$ \\
$x_{1}(n) x_{2}(n) x_{2}^{*}(n)$ & $x_{2}(n) x_{3}(n) x_{3}^{*}(n)$ & $x_{2}(n) x_{3}(n) x_{2}^{*}(n)$ \\
\hline
\end{tabular}

The symbol * denotes the complex conjugate operator. the IMUX and OMUX filters. Secondly, no memory is required for the third nonlinear order terms, and finally, that all fifth-order terms were ruled out from the selected basis functions.

The basis selection process can be made using other models instead of the MIMO Volterra; for instance, memory polynomials [5] or phase-aligned models [47]. However, the MIMO Volterra model subsumes [5] and [47]; in consequence, the results from the basis selection are not affected by this initial model choice. Although the basis selection complexity is high, the overall complexity of the mitigation technique is dominated by the feed forward model propagation rather than the basis selection process [48]. This is due to the slow channel changes ('diurnal' as commonly stated in satellite applications); thereby enabling to perform the basis selection process offline.

\section{Link performance}

From the system deployment perspective, DPD and EQ are different techniques, while an important difference between these techniques is that the OBO, HPA operating point, is affected by the DPD. The OBO point is affected due to the change in the excitation signal caused by the DPD; the change depends on the statistical properties of the input signal, the level of nonlinear distortions (i.e., the level of aggregate OBO), and the DPD technique. Figure 7 shows the loss in $\mathrm{OBO}$ versus the operating aggregate $\mathrm{OBO}$ at the satellite transponder when using the DPD technique previously described. The OBO loss is the difference in aggregate OBO between the DPD case and 


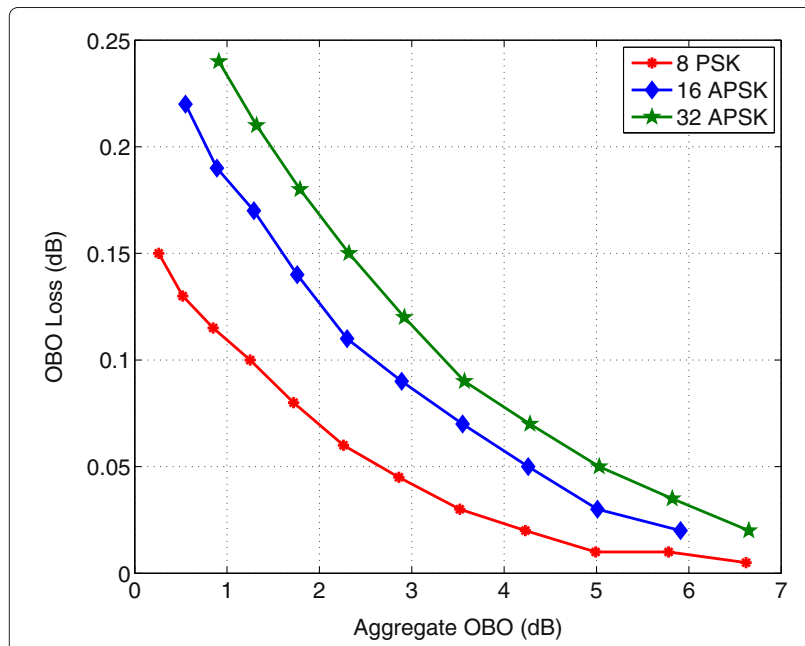

Figure 7 Average loss in $\mathrm{OBO}$ at the satellite transponder due to the use of the proposed multicarrier data DPD.

without DPD for a fixed IBO. Note that for lower values of $\mathrm{OBO}$, the effect is larger as higher levels of distortions arise in the channel. The OBO loss depends on the modulation format as 16/32 APSK are sensitive to nonlinear effects. Finally, note that for large values of OBO, the losses decrease as the channel behaves more linearly and the DPD effect becomes minor. From a power efficiency perspective, the loss in $\mathrm{OBO}$ is undesirable and it negatively affects the link performance. However, the DPD algorithm proposed in this paper causes OBO losses of a few tenths of a $d B$ (cf. Figure 7). Further, the loss in $\mathrm{OBO}$ is more than compensated by the gains obtained in $E_{s} / N_{o}$, leading to enhanced TD as will be shown below.

Figure 8 shows the TD of the three-carrier satellite link as a function of OBO in the HPA; for 16 APSK using a $\frac{3}{4}$ code rate, the outer and inner carriers are presented in Figure $8 \mathrm{a}$ and Figure $8 \mathrm{~b}$, respectively. In this comparison, solely data mitigation techniques are presented. The TD of only one outer carrier is shown due to symmetry. In Figure 8 , the benefit of applying nonlinear mitigation techniques can be observed. Figure 8 includes single carrier data DPD for comparison [2]. Since single carrier predistortion does not include intermodulation products, it is ineffective at combating nonlinear distortions appearing in the multicarrier scenario; consequently, it has poorer performance compared to the proposed multicarrier data predistortion (cf. Figure 8). The proposed data equalizer is compared with the multicarrier interference canceler of [4]. It can be observed from Figure 8 that the proposed

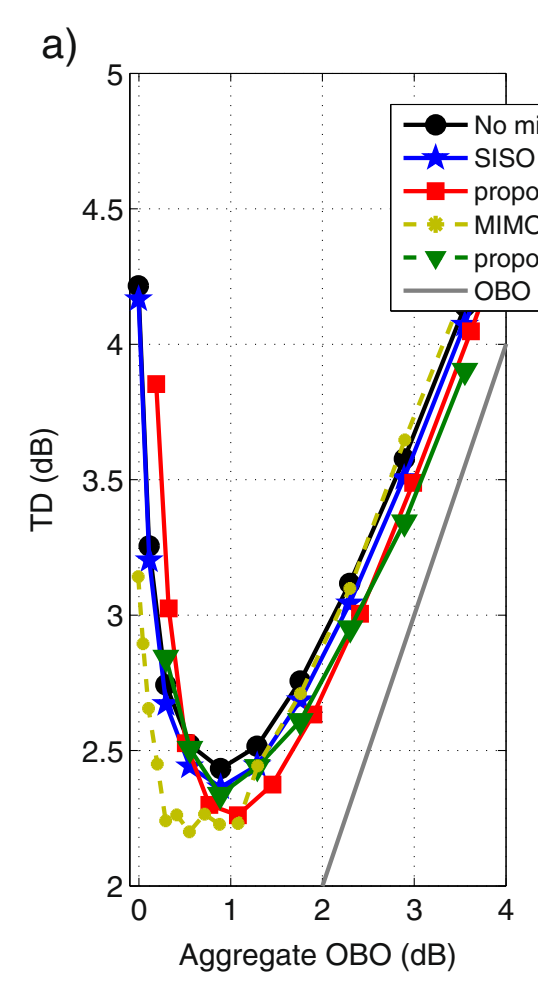

b)

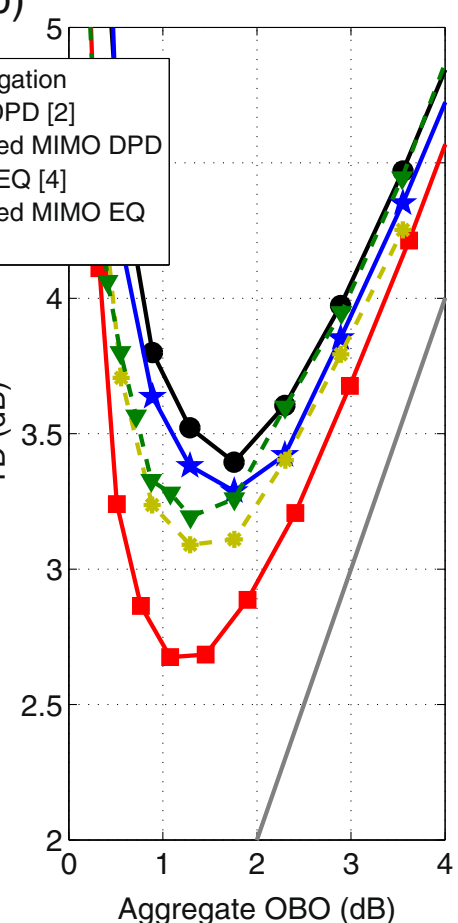

Figure 8 Performance comparison in TD versus aggregate $\mathrm{OBO}$ for data mitigation techniques. The system simulated is a three-carrier satellite link using 16 APSK $\frac{3}{4}$ in every carrier. (a) Outer carrier. (b) Inner carrier. Both proposed multicarrier DPD and EQ use the basis functions indicated in Table 2. 
EQ scheme has some losses compared to the reference receiver technique of [4]. In fact, the multiple carrier turbo Volterra equalizer [4] relies on the powerful, but highly complex, iterative interference cancellation and decoding paradigm. On the other hand, our proposed low complexity equalization does not include any data decoding nor interference cancellation. Further, the proposed EQ has the same complexity of the proposed DPD technique, which is dual for the gateway. The complexity of the DPD is further discussed in the following sections. As seen Figure 8 , the TD performance depends on the carrier location, as the inner carrier exhibits significantly more degradation than the outer ones. While the benefit of applying multicarrier over single carrier mitigation techniques is evident at the inner carrier, the performance gain is lower in the outer carriers where memory effects (ISI) dominate over ACI.

The investigated multicarrier mitigation techniques show different performance in Figure 8 . The differences are due to the effects of the receiver noise, both in the identification stage as well as during operation. While the DPD uses a dedicated receiver that can be designed to have low noise, the equalization has to operate on-the-fly at the receiver with higher levels of noise as in standard operation mode. Note that the equalizer has to operate on received signals which are corrupted by AWGN. Since the equalization operation is nonlinear in the received symbols, the subsequent elements of the chain are affected by a nonlinear function of the front-end noise. This aspect is missing in the DPD and hence it provides further performance enhancement (cf. Figure 8).

Figure 9 compares the TD performance when using three different DPD schemes: i) the multicarrier data memory polynomial DPD [5]; ii) the proposed multicarrier data DPD in Table 2; iii) single carrier waveform signal DPD [19]. The outer and inner carriers are presented in Figure 9a and Figure 9b, respectively. The multicarrier data level memory polynomial [5] has 216 polynomial basis and shows similar TD performance as the proposed data level predistorter which uses only 17 basis functions. Hence, for the multicarrier data DPD schemes, there is a large complexity reduction of the predistorter with no degradation in TD performance. Notice that the single carrier DPD [19] is not a multicarrier technique and presents a larger complexity when compared to data schemes.

Table 3 compares the computational complexity of the different DPD schemes. The complexity is measured with number of floating point operations per second (FLOPS). That is, combining the predistorter computation (number of operations) with its digital processing rate to produce a metric of the computational resources used per second. The predistorter computation refers to the resources required to propagate one symbol from the input to the

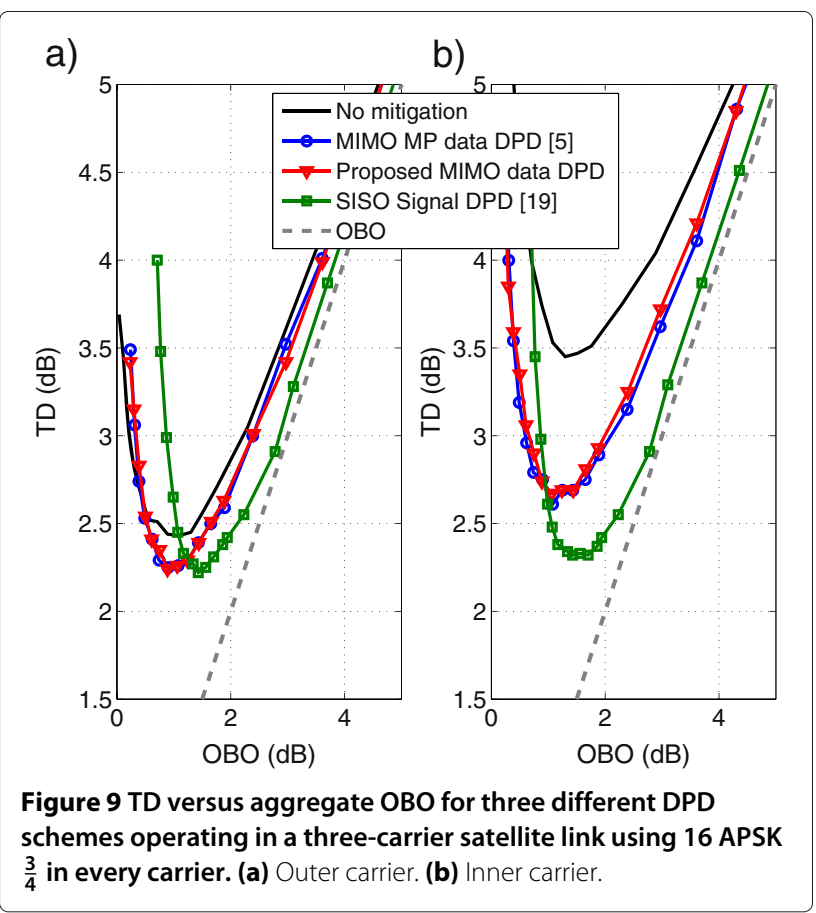

output of the predistorter, since this is the dominating factor for complexity in digital mitigation schemes [48]. From the system settings described in Table 1 , the proposed data predistorter operates at 7 Msamples/s performing: 35 complex multiplications (27 from the third nonlinear order and 8 from the linear part) and 14 complex additions. On the other hand, the signal DPD solution proposed in [19] operates at 183.75 Msamples/s, resulting from the IMUX bandwidth of $26 \mathrm{MHz}$ increased by a 7th nonlinear order considered. At this higher sampling rate, the signal DPD [19] performs at least 150 complex multiplications and 157 complex additions. Hence, the proposed data mitigation technique is much lower in complexity compared to signal DPD, which in turn can be unfeasible in large bandwidth satellite systems due to the increase of processing rate. Table 3 indicates the number of basis functions used in each predistorter, the digital processing rate required, and finally the FLOPS calculated according to [48] scaled by the digital processing rate of each predistorter technique. From Table 3, the proposed predistorter is the one with the lowest computational resources required. Further, the memory polynomial multicarrier data DPD [5] has more than ten times FLOPS than the proposed predistorter with the same TD performance. Finally, signal DPD scheme [19] offers the best TD performance (c.f Figure 9). However, signal DPD has a larger OBO loss when compared to other mitigation schemes, which results in optimal TD performance at larger OBO. Furthermore, signal DPD is not a MIMO (multicarrier) solution, thereby requiring different 
Table 3 Comparison of DPD schemes

\begin{tabular}{lcccc}
\hline & \multicolumn{2}{c}{ MIMO (multicarrier) } & & SISO (single carrier) \\
\cline { 2 - 4 } Scheme & 3 & Memory polynomial [5] & 3 & Signal DPD [19] \\
\hline \# carriers & 17 & 216 & 1 \\
\hline \# basis & $T_{r}$ & $T_{r}$ & 52 \\
\hline Digital processing & 7 & 7 & $7 \times 3(1+\rho) T_{r}$ \\
rate (Msamples/s) & 1,750 & 19,280 & 183.75 \\
\hline FLOPS $\left(10^{6}\right)$ & & 163,400 & 1 \\
\hline
\end{tabular}

hardware architecture and a frequency mask to fulfill the stringent control of the out-of-band emissions from the gateway. Moreover, signal DPD requires more than 90 times the amount of FLOPS compared to the proposed solution. The increase in the number of FLOPS from the proposed DPD scheme to the memory polynomial [5] is due to the increase in the number of operations (from 17 to 216 basis functions). However, the increase in number of FLOPS between the multicarrier and single carrier DPD schemes is mainly due to increase of the digital processing rate. In particular, the large amount of FLOPS in signal DPD [19] is due to its high digital processing rate, as it operates $173.75 / 7 \approx 26$ times faster than the other DPD schemes studied. This increased digital processing rate is required to faithfully represent the nonlinear operators which enlarge the available input bandwidth, thereby requiring a larger digital processing rate.

Extensive simulations were performed for several modulation formats and code rates in order to determine the performance of the mitigation techniques. In all presented results, the TD was obtained by sweeping the signal-tonoise ratio $\left(E_{s} / N_{o}\right)$ of the received symbols until the target BER of $10^{-5}$ was achieved. The TD showed trends similar to Figure 8. In general, the level of TD is affected by the modulation format and the code rate. First, the TD increases as the number of constellation symbols increases; this can be explained by the higher sensitivity of the higher modulation formats resulting in higher levels of TD. Secondly, TD decreases as the ratio of redundancy and information increases in the code rate; this is expected as the error correcting property of the decoder improves with redundancy [42].

In a multicarrier scenario, the optimal (minimum) TD may appear at different OBO levels for different carriers. This effect could complicate the task of choosing the operating point of the HPA, leading to compromises between different carriers. For instance, in Figure 8 for the case with no mitigation techniques, the optimal TD of the inner and outer carrier occurs at around 1.8 and 1 $\mathrm{dB}$ aggregate $\mathrm{OBO}$, respectively. The use of multicarrier mitigation techniques also helps reducing the difference in OBO between the optimal TD of different carriers and consequently the need for compromising between them, as depicted in Figures 8 and 9.

\section{Sensitivity results}

Total degradation shows a convex behavior with respect to the OBO (cf. Figure 8 ). TD is sensitive to the transmission scheme, especially to the modulation and code rate. In Figure 10, we show the trend of the optimal TD per carrier with respect to the code rate for a fixed modulation of 16 APSK.

As expected, the degradation increases with the code rate, while at the same time, relative gains of the applied DPD and equalization techniques increase as well. This is intuitively justifiable noticing that when a strong code is applied, the channel is typically in a noise-limited regime and the nonlinear mitigation techniques have a minor effect on performance. On the other hand, when a weak

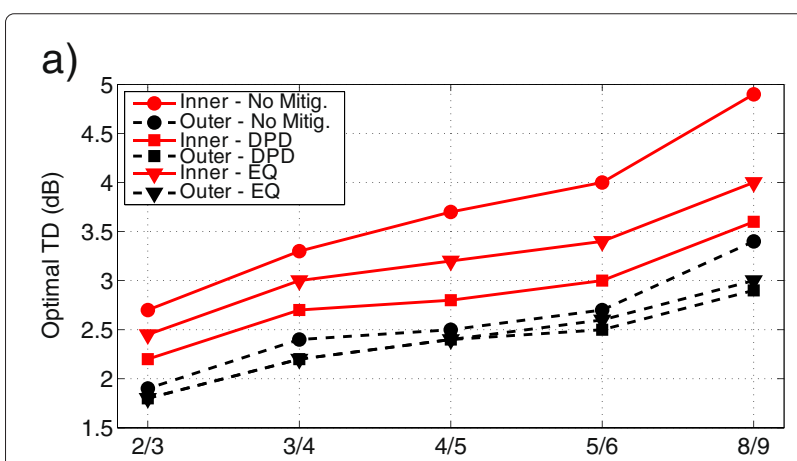

b)

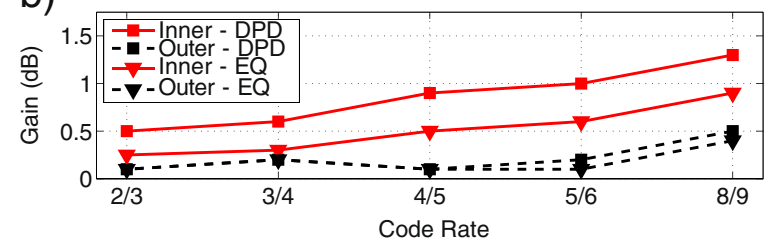

Figure 10 Optimal TD versus code rate and its improvement when using DPD or EQ. (a) Variation of optimal TD versus code rate in a three-carrier 16 APSK simulation. (b) Improvement (gain) in TD using a mitigation technique. Improvement obtained using the data in (a). 
code rate is applied, the channel is in an interferencelimited regime and the benefits of mitigation techniques become more relevant.

Figure 11 shows the optimal TD in the inner carrier (worst-case performance) of the three-carrier system as a function of the spectral efficiency. Simulations of different modulation formats and code rates were performed. For every modulation format considered, the minimum and maximum code rate settings of [3] have been included in this simulation. The spectral efficiency is proportional to $R \log _{2}(S)$, with $R$ being the code rate and $S$ the number of constellation symbols in the modulation format.

In Figure 11, we notice that for a given TD, the channel spectral efficiency can be significantly increased by applying mitigation techniques and changing the modulation format.

\section{Conclusions}

This paper studied the use of nonlinear mitigation techniques to the situation where multiple carriers were amplified by a single HPA in a satellite transponder. The mitigation techniques, predistortion (DPD), and equalization (EQ), are described in the framework of MIMO Volterra series. However, the large number of coefficients required in the mitigation techniques limits their practical applicability. In this paper, a basis pursuit approach is employed using the LASSO algorithm to reduce the complexity of the mitigation technique yielding models with fewer number of coefficients that keep limited modeling error. In general, the model complexity of mitigating techniques grows with increasing number of carriers and hence the need for complexity reduction is exacerbated in the DPD/EQ models. Although the LASSO method

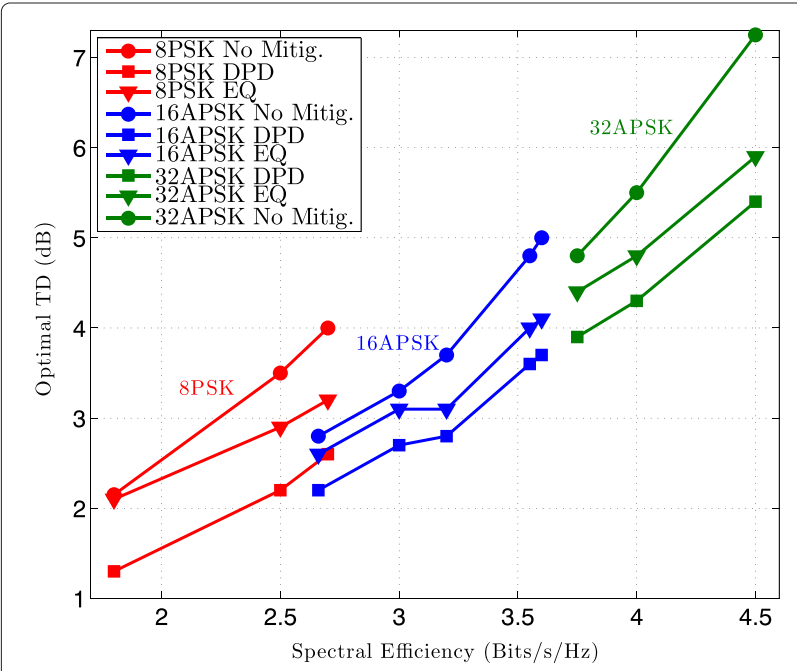

Figure 11 Dependence of the TD on spectral efficiency: three-carrier simulation, inner carrier result reported. described in this paper has been applied in a three-carrier scenario, it can still be used for larger number of carriers. The LASSO approach would also be of benefit in terrestrial applications where signal DPD is used for concurrent multiband HPAs.

The proposed DPD mitigation scheme reduces the complexity (measured as the number of FLOPS) of multicarrier memory polynomial models by a factor of 10 while achieving nearly the same TD performance. On the other hand, single carrier signal DPD achieves better TD performance than the proposed mitigation at an expense of 90 times more FLOPS. Extensive simulations showed that the use of the proposed mitigation techniques is favorable to the overall link performance. Moreover, for a specific $\mathrm{TD}$, the use of these techniques allow to operate the HPA at lower OBO which improves the power efficiency.

It was found that DPD gave better performance than equalization in terms of TD. The explanation of this difference is the level of noise in the received signals. While DPD is estimated using the received data from a reference receiver, which is designed to be low noise, $\mathrm{EQ}$ is evaluated at higher noise levels, from standard receivers. Further, the proposed equalizer is obtained as the channel inverse which prevents the received noise power being used into its estimation procedure and causes a bias in the estimates [49]. Moreover, DPD operates on nearly noiseless data from the transmitter, while the EQ operates on the received noisy data. This implies that in the $\mathrm{EQ}$, the noise at the receiver propagates through a nonlinear compensation scheme, which is missing in the DPD and further explains the different gains in performance.

\section{Abbreviations}

ACl: adjacent channel interference; APSK: amplitude and phase shift keying; AWGN: additive white Gaussian noise; BER: bit error rate; DPD: digital predistortion; DTH: direct-to-home; DVB-S2: Digital Video Broadcasting Satellite - Second Generation; EQ: equalization; GW: gateway; HPA: high-power amplifier; IBO: input back-off; IMD: inter-modulation products; IMUX: input multiplexing; ISI: intersymbol interference; LASSO: least absolute shrinkage and selection operator; LDPC: low-density parity-check; MIMO: multiple input multiple output; OBO: output back-off; OMUX: output multiplexing; SISO: single input single output; SRRC: square root raised cosine; TD: total degradation; TWTA: traveling wave tube amplifier.

\section{Competing interests}

The authors declare that they have no competing interests.

\section{Author details \\ 'Department of Electronics, Mathematics and Natural Sciences, University of Gävle, Kungsbäcksvägen 47, 801-76 Gävle, Sweden. ${ }^{2}$ Department of Signal Processing, The Royal Institute of Technology KTH, Osquldas väg. 10, 100-44 Stockholm, Sweden. ${ }^{3}$ Interdisciplinary Centre for Security, Reliability and Trust (SnT), University of Luxembourg, 4, rue Alphonse Weicker, L-2721, Luxembourg.}

Received: 16 June 2014 Accepted: 4 March 2015

Published online: 24 March 2015

\section{References}

1. A Morello, V Mignone, DVB-S2: The second generation standard for satellite broad-band services. Proc. IEEE. 94(1), 210-227 (2006) 
2. E Casini, R De Gaudenzi, A Ginesi, DVB- S2 modem algorithms design and performance over typical satellite channels. Int. J. Satell. Commun. Netw. 22(3), 281-318 (2004)

3. ETSI Standard, Digital Video Broadcasting (DVB), Second generation framing structure, channel coding and modulation systems for broadcasting, interactive services, news gathering and other broadband satellite applications (DVB-S2). European Telecommunications Standards Institute (ETSI) EN 302307 V1.1.2 (2006-06)

4. BF Beidas, Intermodulation distortion in multicarrier satellite systems: analysis and turbo Volterra equalization. IEEE Trans. Commun. 59(6), 1580-1590 (2011)

5. R Piazza, MR Bhavani Shankar, E Zenteno, D Rönnow, J Grotz, F Zimmer, M Grasslin, Heckmann F, S Cioni, in Proc. 30th AIAA Int. Comm. Satellite, Systems Conf. (ICSSC). Multicarrier digital pre-distortion/equalization techniques for non-linear satellite channels (Ottawa, Canada, 2012)

6. S Benedetto, E Biglieri, Nonlinear equalization of digital satellite channels. IEEE J. Sel. Areas Commun. 1, 57-62 (1983)

7. L Ding, GT Zhou, DR Morgan, Z Ma, JS Kenney, J Kim, Giardina C R, A robust digital baseband predistorter constructed using memory polynomials. IEEE Trans. Commun. 52(1), 159-165 (2004)

8. BF Beidas, R Seshadri, Analysis and compensation for nonlinear interference of two high-order modulation carriers over satellite link. IEEE Trans. Commun. 58(6), 1824-1833 (2010)

9. M Allegue-Martinez, N Kelly, A Zhu, Digital linear pre-compensation technique to enhance predistortion performance in multicarrier DVB-S2 satellite communication systems. Electron. Lett. 50(13), 957-959 (2014)

10. C Eun, EJ Powers, A new Volterra predistorter based on the indirect learning architecture. IEEE Trans. Signal Process. 45(1), 223-227 (1997)

11. M Isaksson, D Wisell, D Rönnow, A comparative analysis of behavioral models for RF power amplifiers. IEEE Trans. Microw. Theory Tech. 54(1), 348-359 (2006)

12. F Langlet, D Roviras, A Mallet, F Castanie, in Proc. Int. Joint Conf. Neural, Networks, (IJCNN). Mixed analog/digital implementation of MLP NN for predistortion, vol. 3 (Honolulu, HI, 2002), pp. 2825-2830

13. S Bouchired, M Ibnkahla, D Roviras, F Castanie, in IEEE Int. Conf. Acoustics, Speech, and Signal, Processing. Equalization of satellite UMTS channels using neural network devices, vol. 5 (Phoenix, AZ, 1999), pp. 2563-2566

14. N Naskas, Y Papananos, Neural-network-based adaptive baseband predistortion method for RF, power amplifiers. IEEE Trans. Circuits Syst. II Exp. Briefs. 51(11), 619-623 (2004)

15. M Isaksson, D Wisell, D Rönnow, Wide-band dynamic modeling of power amplifiers using radial-basis function neural networks. IEEE Trans. Microw. Theory Tech. 53(11), 3422-3428 (2005)

16. R Zayani, R Bouallegue, D Roviras, in IEEE 21st Int. Symp. Personal, Indoor and Mobile Radio Communications (PIMRC). Crossover neural network predistorter for the compensation of crosstalk and nonlinearity in MIMO OFDM systems (Istanbul, Turkey, 2010), pp. 966-970

17. R Piazza, B Shankar, B Ottersten, in IEEE Int. Conf. Communications (ICC) Multicarrier LUT-based data predistortion for non-linear satellite channels (Sydney, NSW, 2014), pp. 4319-4324. doi:10.1109/ICC.2014.6883999

18. T Deleu, M Dervin, F Horlin, in IEEE Int. Conf. Communications (ICC). Low complexity block pre-distortion of a multi-carrier non-linear satellite channel (Sydney, NSW, 2014), pp. 4325-4330. doi:10.1109/ICC.2014.6884000

19. N Kelly, A Zhu, TJ Brazil, in Proc. 31th AlAA Int. Comm. Satellite, Systems Conf. (ICSSC). Digital predistortion feasibility studies for multicarrier satellite communication systems (Florence, Italy, 2013)

20. GE Corazza, Digital Satellite Communications, Chapter 8. (Springer, New York, USA, 2007)

21. J Kim, Roblin P, D Chaillot, Z Xie, A generalized architecture for the frequency-selective digital predistortion linearization technique. IEEE Trans. Microw. Theory Tech. 61(1), 596-605 (2013)

22. SA Bassam, M Helaoui, FM Ghannouchi, Crossover digital predistorter for the compensation of crosstalk and nonlinearity in MIMO transmitters. IEEE Trans. Microw. Theory Tech. 57(5), 1119-1128 (2009)

23. S Amin, PN Landin, P Händel, D Rönnow, Behavioral modeling and linearization of crosstalk and memory effects in RF MIMO transmitters. IEEE Trans. Microw. Theory Tech. 62(4), 810-823 (2014)

24. SA Bassam, W Chen, M Helaoui, FM Ghannouchi, Z Feng, Linearization of concurrent dual-band power amplifier based on 2D-DPD technique. IEEE Microw. Wireless Compon. Lett. 21(12), 685-687 (2011)
25. J Harmon, SG Wilson, in Military Comm. Conf., (MILCOM). Remote satellite amplifier predistortion using the indirect learning architecture (San Jose, CA, 2010), pp. 335-340. doi:10.1109/MILCOM.2010.5680352

26. G Colavolpe, Piemontese A, in Proc. IEEE Global Telecommun. Conf.; Houston, USA. Novel SISO detection algorithms for nonlinear satellite channels (IEEE, Piscataway, 2011)

27. R Tibshirani, Regression shrinkage and selection via the LASSO. J. R. Statist. Soc. B. 58(1), 267-288 (1994)

28. V Kekatos, GB Giannakis, Sparse Volterra and polynomial regression models: recoverability and estimation. IEEE Trans. Signal Process. 59(12), 5907-5920 (2011)

29. AAM Saleh, Frequency-independent and frequency-dependent nonlinear models of TWT amplifiers. IEEE Trans. Commun. 29(11), 1715-1720 (1981)

30. M Schetzen, The Volterra and Wiener Theories of Nonlinear Systems. (Wiley \& Sons, New York, 1980)

31. LM Li, SA Billings, Generalized frequency response functions and output response synthesis for mimo non-linear systems. Int. J. Control. 79(1), 53-62 (2006)

32. ZK Peng, ZQ Lang, SA Billings, Non-linear output frequency response functions for multi-input non-linear Volterra systems. Int. J. Control. 80(6), 843-855 (2007)

33. AK Swain, SA Billings, Generalized frequency response function matrix for MIMO non-linear systems. Int. J. Control. 74(8), 829-844 (2001)

34. DR Hummels, R Gitchell, Equivalent low-pass representations for bandpass Volterra systems. IEEE Trans. Commun. 28(1), 140-142 (1980)

35. SM Kay, Fundamentals of Statistical Signal Processing: Estimation Theory. (Prentice-Hall, Inc, Upper Saddle, River, NJ, USA, 1993)

36. P Stoica, B Friedlaner, Söderström T, On the parsimony principle. Int. J. Control. 36(3), 409-418 (1982)

37. K Shi, P Shi, Adaptive sparse volterra system identification with $\ell_{0}$-norm penalty. Signal Process. 91(10), 2432-2436 (2011)

38. N Kalouptsidis, G Mileounis, B Babadi, V Tarokh, Adaptive algorithms for sparse system identification. Signal Process. 91(8), 1910-1919 (2011)

39. H Zou, The adaptive LASSO and its oracle properties. J. Am. Statist. Assoc. 101(476), 1418-1429 (2006)

40. J Huang, P Breheny, S Ma, A selective review of group selection in high-dimensional models. Statist. Sci. 27(4), 481-499 (2012)

41. M Aloisio, E Casini, A Ginesi, Evolution of space traveling-wave tube amplifier requirements and specifications for modern communication satellites. IEEE Trans. Electron Devices. 54(7), 1587-1596 (2007)

42. RG Gallager. Low density parity-check codes (PhD thesis, MIT Press Cambridge MA, 1963)

43. M Grant, S Boyd, Graph implementations for nonsmooth convex programs. Recent Advances in Learning and Control. Lecture Notes in Control and Information Sciences. (V Blondel, S Boyd, H Kimura, eds.) (Springer-Verlag Limited, New York, 2008)

44. M Grant, S Boyd, CVX: Matlab Software for Disciplined Convex Programming, version 2.1. http://cvxr.com/cvx, Accessed 25/March/2015

45. DG Luenberger, Microeconomic Theory. (McGraw-Hill, New York, 1995)

46. A Wächter, LT Biegler, On the implementation of an interior-point filter line-search algorithm for large-scale nonlinear programming. Math. Program. 106(1), 25-57 (2006)

47. M Younes, Kwan A, M Rawat, FM Ghannouchi, Linearization of concurrent tri-band transmitters using 3-D phase-aligned pruned Volterra model. Microw. Theory Tech. IEEE Trans. 61(12), 4569-4578 (2013)

48. A Tehrani, H Cao, S Afsardoost, T Eriksson, M Isaksson, C Fager, A comparative analysis of the complexity/accuracy tradeoff in power amplifier behavioral models. IEEE Trans. Microw. Theory Tech. 58(6), 1510-1520 (2010)

49. S Amin, E Zenteno, PN Landin, D Ronnow, M Isaksson, P Handel, in Swedish Communication Technologies Workshop, (Swe-CTW). Noise impact on the identification of digital predistorter parameters in the indirect learning architecture (Lund, Sweden, 2012), pp. 36-39 